\title{
UK medical selection: lottery or meritocracy?
}

Authors: Benjamin HL Harris, $\mathrm{A}$ Jason L WalshB and Simon LammyC

Clinical Medicine 2015;15:40-6.

A correction to Box 1 was missed under 'Most likely score if guessed'. The corrected box is printed below.

\section{Box 1. Why guessing pays dividends.}

Format:

SJT paper $=70$ questions in 2 hours 20 minutes

60 questions are 'live' and 10 'pilot' questions are not included in the participant's final score

Approximately two-thirds of questions are ranking questions and one-third are 'select three from eight' MCQs.

Job application score scaling method:

SJT converted score $=\left(S J T\right.$ raw score $\left.{ }^{A} \times 0.154\right)-90.1$

Formula from the UK Foundation Programme Office

Ranking question:

> Maximum raw score per question $=20 / 20$ marks

- $(20 \times 0.154)=3.08$ job application marks

$>$ Most likely score if guessed $=12 / 20$

- $(12 \times 0.154)=1.848$ job application marks

> No attempt $=0$ job application marks

'Select three from eight' MCQs:

> Maximum raw score per question $=12 / 12$ marks

- $(12 \times 0.154)=1.848$ job application marks

$>$ Average raw score if guessed $=4.5 / 12$

- $(4.5 \times 0.154)=0.693$ job application marks

> Minimum score per attempt/no attempt $=0 / 12$

AStrictly test equated raw score across sittings.

Available online at www.foundationprogramme.nhs.uk/pages/home/ how-to-apply/SJT-FAQs\#answer379K

$\mathrm{MCQ}=$ multiple choice questions; $\mathrm{SJT}=$ situational judgement test. 